\title{
1D and 3D defect characterization in IR thermographic NDT
}

\author{
by V.P. Vavilov, A.O. Chulkov
}

Tomsk Polytechnic University, School of Nondestructive Testing, Lenin Av., 634050, Tomsk, Russia, vavilov@tpu.ru

\begin{abstract}
Defect characterization is a specific procedure of IR thermographic nondestructive testing (NDT) which follows a stage of defect detection. Both procedures can be reference-free or introduce a reference area defined automatically or by the thermographer. The typical reference-free concepts are Pulse Phase Thermography (PPT), Principal Component Analysis (PCA), Thermographic Signal Reconstruction (TSR) and some others. However, by choosing a reference point close to a suspicious (allegedly defect-linked) zone one may consider subtle differences between defect and non-defect areas. Such differences are typically related to differential temperature signals which, in their turn, can be converted into phase shift signals, effusivity variations, etc. In this study, two approaches, namely, analytical formulas and a neural network, have been applied to characterize defects in composites.
\end{abstract}

\section{Introduction}

Thermal nondestructive testing (TNDT) is a good illustration to the classification of heat conduction problems for direct and inverse. In the direct formulation, temperature distributions are calculated by knowing test geometry, including all parameters of hidden defects, and this process is called modeling. Inverse problems assume that temperature distributions are measured experimentally and some geometrical parameters are to be inversely retrieved. This concept represents practical (experimental) NDT oriented toward defect detection and characterization. TNDT problems are multi-parametric and based on the heat conduction equation, therefore, immediate inversion of experimental results, as a rule, is impossible. Mathematically, the most general approach is based on the analysis of functional residuals produced by comparing experimental and theoretical data. This approach requires searching global extremums of a respective functional formulated in a multidimensional space of TNDT features; in TNDT publications, this method is often called non-linear fitting. In practice, some simple solutions to inverse TNDT problems can be found by fitting theoretical direct solutions with some inversion formulas. To do this, both analytical and numerical direct solutions can be analyzed. The structure of respective inversion formulas can be prompted by direct solutions. Numerical coefficients in inversion formulas are typically obtained by applying a least-square technique; the corresponding options are available in some commercial software packages, such as Mathematica, Matlab, etc.

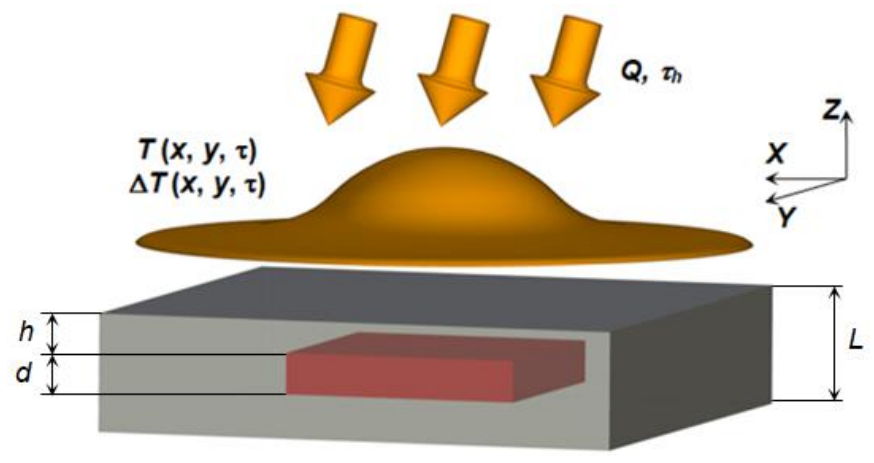

Fig. 1: Scheme of defect thermal characterization.

Figure 1 shows schematically a physical model of defect thermal characterization. A solid body by the thickness $L$ characterized by the thermal conductivity $K$ and diffusivity $\alpha$ is heated with the thermal flux $Q$ during the time $\tau_{\mathrm{h} .}$ A subsurface defect disturbs a regular flow of heat in the body bulk thus producing the corresponding local temperature change $\Delta T=T_{d}-T_{n d}$ (" $d$ " and " $n d$ " specify defect and non-defect areas) that is a function of surface coordinates $x, y$ and time $\tau$. In fact, the so-called data cube $T(x, y, \tau)$ supplies inputs to solving a respective inverse TNDT problem. The outputs are: defect depth $h$, defect lateral dimensions $d_{x}$ and $d_{y}$ and defect thickness $d$, or thermal resistance $R_{d}=d / K_{d}$. It is important noting that estimates of $d_{x}$ and $d_{y}$ are the only ones which can be obtained by directly analyzing surface temperature indications [1]. In fact, defect lateral shape and size can be estimated, often with a reasonable accuracy, by visually evaluating surface temperature anomalies. The most challenging is the evaluation of $h$ and $d$ (or $R_{d}$ ). Many algorithms for characterizing $h$ and $R_{d}$ have been proposed elsewhere [2-7].

However, available defect characterization algorithms are mostly one-dimensional (1D), i.e. they are based on 1D heat conduction solutions thus discarding lateral size of defects. At Tomsk Polytechnic University, a ThermoFit Pro program was developed to process both experimental and synthetic IR image sequences. Along with standard data 
processing algorithms, such as filtration, PPT, PCA, normalization, wavelet transform, etc., the program includes two defect characterization options which are based on polynomial fitting of multi-parametric analytical (1D) and numerical three-dimensional (3D) heat conduction solutions; in the latter case, inversion formulas are corrected by apparent defect lateral dimensions. The proposed algorithms are fast and robust unlike those involving non-linear fitting.

In this study, both 1D and 3D algorithms have been applied to the inspection of carbon fiber reinforced plastic (CFRP) composite. The 1D defect characterization algorithm applied was analytical (ThermoFit Pro software), and the 3D algorithm was implemented in an artificial neural network (NN) trained to evaluate defect size and depth.

\section{The sample and testing procedure}

The $230 \times 190 \times 16 \mathrm{~mm}$ test sample was made of CFRP and contained 8 bottom-hole defects with the radius of $20 \mathrm{~mm}$ (figure 2a). The one-sided active thermal nondestructive testing (TNDT) of the sample was conducted in two different ways. For the first test procedure (figure $2 \mathrm{~b}$ ), the experimental setup was assembled by using the halogen lamp $(2 \mathrm{~kW})$ placed in front of the sample that is a classical one-sided approach. In the second test procedure (figure $2 \mathrm{c}$ ), the sample was moving at a constant speed under the linear halogen lamp heater $(2 \mathrm{~kW})$ by means of a step motor; thus the scanning TNDT approach was realized. In both cases, the duration of the heat excitation was $10 \mathrm{~s}$. To record temperature distributions, an infrared (IR) module Optris PI 640 was used. In the case of scanning procedure, a sample was moving with the speed of $5 \mathrm{~mm} / \mathrm{s}$ across the heated area with the width of $50 \mathrm{~mm}$ thus ensuring $10 \mathrm{~s}$-long heating of each sample point. The sample surface temperature was recorded at the cooling stage within the time period from 20 to $85 \mathrm{~s}$ after heating stopped. The standard TNDT parameters have been determined for all defects, namely, optimal observation times $\tau_{m}$ and maximum temperature contrasts $C=\left(T_{d}-T_{n d}\right) / T_{n d}$ (see table 1$)$. Note that test parameters, such as the movement speed $(5 \mathrm{~mm} / \mathrm{s})$, acquisition frame rate $(4.6 \mathrm{~Hz})$ and the width of a single scanned line $(1.09 \mathrm{pix} / \mathrm{mm})$ have been carefully chosen to ensure the synthesis of resulting IR images without under- and overlapping of scanned lines.

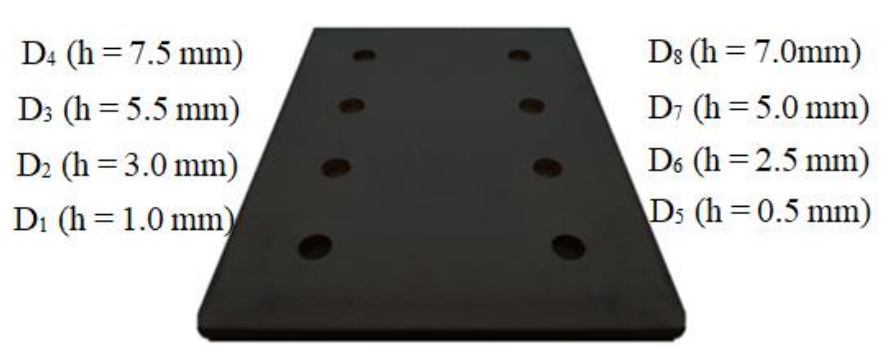

(a)
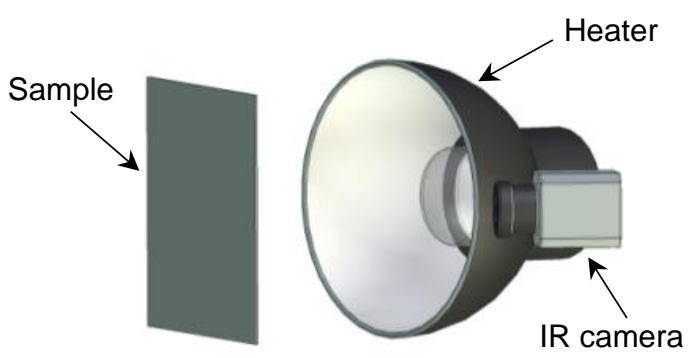

(b)

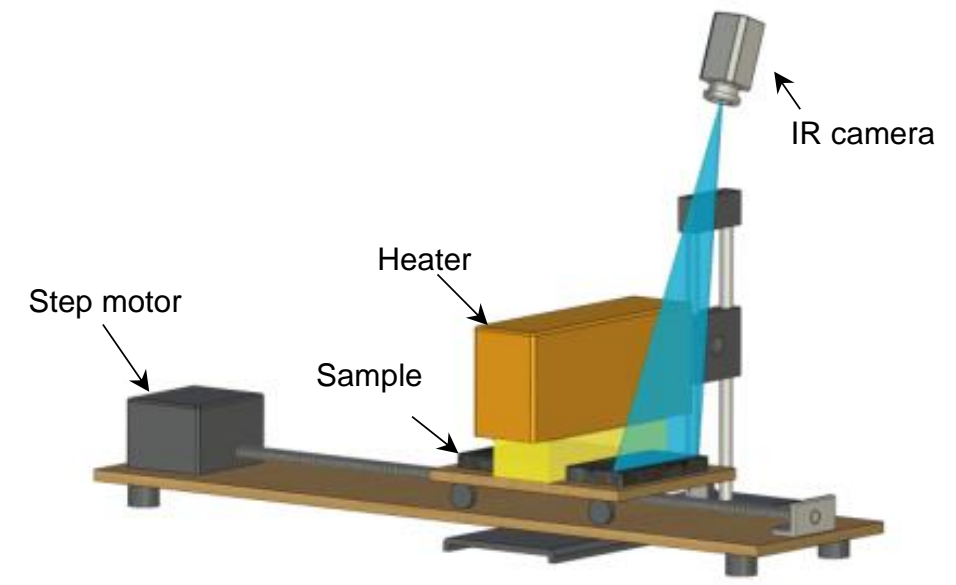

(c)

Fig. 2: One-sided thermal nondestructive testing of CFRP sample:

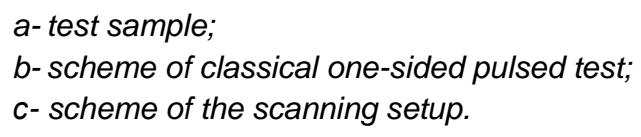


Table 1: Optimal observation time for defects D1-D8 (Fig. 8a).

\begin{tabular}{|c|c|c|c|c|c|c|c|c|}
\hline Defect & $\mathrm{D}_{1}$ & $\mathrm{D}_{2}$ & $\mathrm{D}_{3}$ & $\mathrm{D}_{4}$ & $\mathrm{D}_{5}$ & $\mathrm{D}_{6}$ & $\mathrm{D}_{7}$ & $\mathrm{D}_{8}$ \\
\hline $\mathrm{C}$ & 2.07 & 1.17 & 0.71 & 0.26 & 1.88 & 1.08 & 0.54 & 0.22 \\
\hline$\tau_{\mathrm{m}}, \mathrm{s}$ & 24.0 & 47.5 & 72.0 & 89.0 & 21.1 & 47.0 & 69.0 & 85.0 \\
\hline
\end{tabular}

Test results obtained by using both procedures are given in figure 3 . All hidden defects are seen (figure 3a). Figure $3 b$ illustrates a principle of image reconstruction which was done by synthesizing a resulting image column by column (note that columns were perpendicular to the sample movement direction).

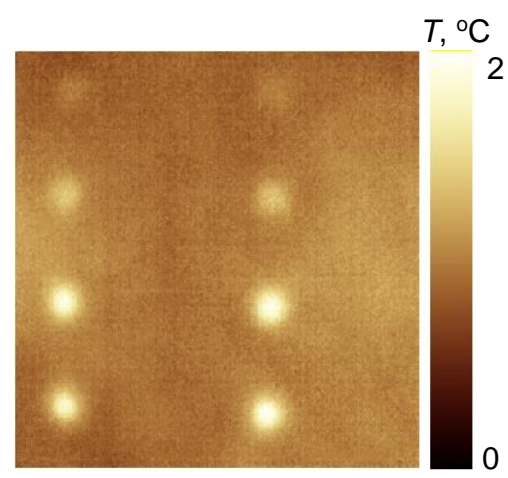

(a)

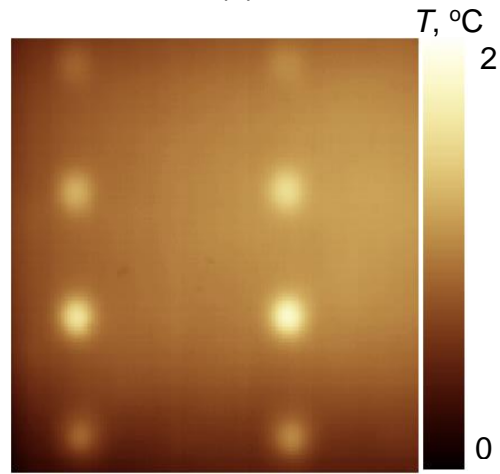

(c)

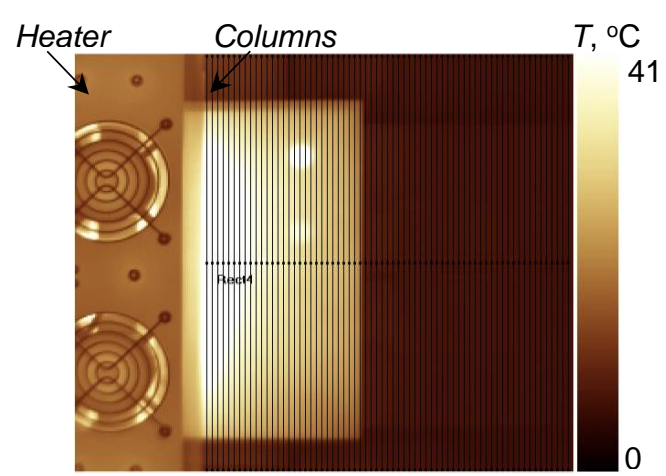

(b)

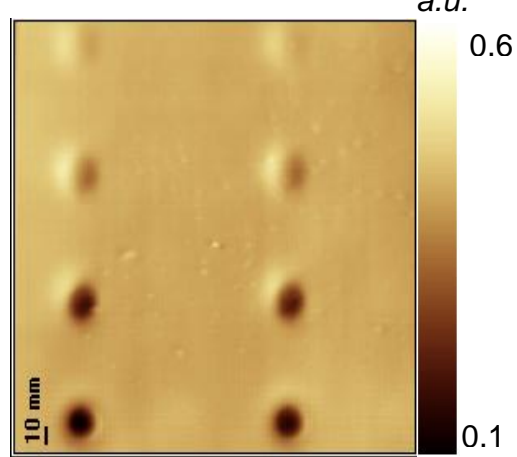

(d)

Fig. 3: TNDT results for different observation times:

a- result of classical one-sided procedure, raw image, test time $52 \mathrm{~s}$,

b- sample image during the scanning,

$c$ - resulting image obtained in the scanning procedure, test time $52 \mathrm{~s}$,

$d$ - image of 1-st derivative (TSR method, scanning procedure), test time $52 \mathrm{~s}$.

\section{Defect characterization}

\subsection{D analytical approach}

In the pulsed procedure, the optimal heating time of $10 \mathrm{~s}$ was chosen empirically to ensure detection of all defects, and the example of an "optimal" image is shown in figure $4 \mathrm{a}$. The sample excess temperature was about $2^{\circ} \mathrm{C}$, and each raw sequence of $\mathrm{IR}$ images was converted into the corresponding sequence of running contrast $C$ images (figure 4b). A chosen reference point is indicated in figure 4a. Respectively, each "contrast" image was processed to calculate images of maximum running contrast $C$ (maxigram) and times of its appearance $\tau_{m}$ (timegram). Afterwards, these parameters were used for determining defect depth $h$ and defect thermal resistance $R_{d}=d / K_{d}$, where $d$ is the defect thickness $[\mathrm{m}]$ and $K_{d}$ is the defect conductivity $\left[\mathrm{W} \cdot \mathrm{m}^{-1} \cdot \mathrm{K}^{-1}\right]$. The analytical inversion formulas implemented in the ThermoFit Pro software for the evaluation of defect parameters in composites are as follows.

If a maximum of $C$ occurs within the heat pulse $\left(\tau \leq \tau_{h}\right)$ :

$$
\begin{gathered}
h[m]=0.407\left(a \tau_{m}\right)^{0.489}\left(1-\frac{K_{d}}{K}\right)^{0.190}\left(\frac{L}{K}\right)^{0.0788} F o_{h}^{0.121} C^{-0.287} \\
R_{d}\left[m^{2} K W^{-1}\right]=1.239\left(a \tau_{m}\right)^{0.404}\left(1-\frac{K_{d}}{K}\right)^{0.0438}\left(\frac{L}{K}\right)^{0.119} F o_{h}^{-0.168} C^{0.226} .
\end{gathered}
$$


If a maximum of $C$ occurs after the heat pulse $\left(\tau>\tau_{h}\right)$ :

$$
\begin{gathered}
h[m]=0.708\left[a\left(\tau_{m}-\tau_{h}\right)\right]^{0.456}\left(1-\frac{K_{d}}{K}\right)^{0.285}\left(\frac{L}{K}\right)^{0.0536} \mathrm{Fo}_{h}^{0.0523} \mathrm{C}^{-0.151}, \\
R_{d}\left[m^{2} K W^{-1}\right]=4.368\left[a\left(\tau_{m}-\tau_{h}\right)\right]^{0.343}\left(1-\frac{K_{d}}{K}\right)^{1.265}\left(\frac{L}{K}\right)^{0.286} \mathrm{Fo}_{h}^{-0.0530} \mathrm{C}^{0.426} .
\end{gathered}
$$

The equations above have been obtained by inverting plentiful direct solutions of a number of defect situations in a wide range of composite materials. The structure of these equations was prompted by some classical heat conduction solutions, such as $=\sqrt{\alpha \tau}$, and authors' experience. However, if needed, they could be précised for a narrower range of materials by more carefully choosing the numerical coefficients. The practical experience of using these equations has shown that the accuracy in determining $h$ is about $15-30 \%$ for different types of composites, while for $R_{d}$ the accuracy drops down to $40-60 \%$. The characterization algorithm described is simple and robust allowing to produce images of defect depth ("depthgrams") and thermal resistance.

The corresponding images are presented in figures 4 to exhibit the following peculiarities of defect thermal characterization. The depthgram in figure $4 \mathrm{c}$ shows depths of particular defects with accuracy from 4 to $23 \%$. The image of defect thermal resistance in figure $4 d$ shows defects in more or less the same color thus prompting that they are characterized by the same thermal resistance. The pixel values in the defect areas correspond to $R_{d^{\sim}} 0.12 \mathrm{~m}^{2} \cdot \mathrm{K}^{\mathrm{W}} \mathrm{W}^{-1}$. Respectively, this produces the estimate of defect thickness of $8.5 \mathrm{~mm}$ if to assume that air conductivity in thin gaps is $0.07 \mathrm{~W} \cdot \mathrm{m}^{-1} \cdot \mathrm{K}^{-1}$, even if the depth of bottom hole is different. This fact is explained by the known saturation of the $\Delta T(d)$ dependence. The estimates above illustrate that thermal characterization of defect thickness is less accurate than that of defect depth. It is worth noting that the images in figures $4 \mathrm{c}$, $\mathrm{d}$ have been produced with an amplitude threshold of $5 \%$ by $C$ values. This allowed to obtain values of $h=16 \mathrm{~mm}$ and $R_{d}=0$ in non-defect areas.

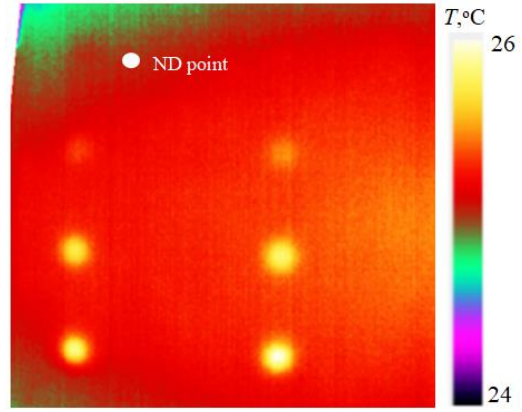

(a)

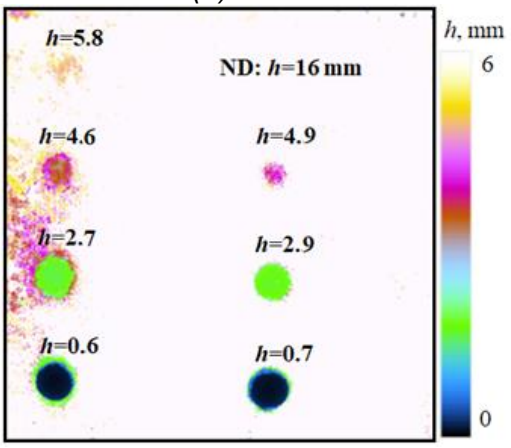

(c)

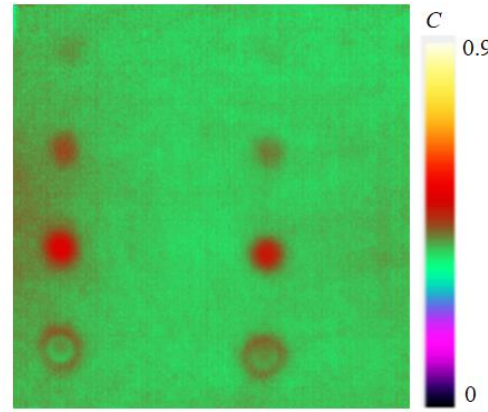

(b)

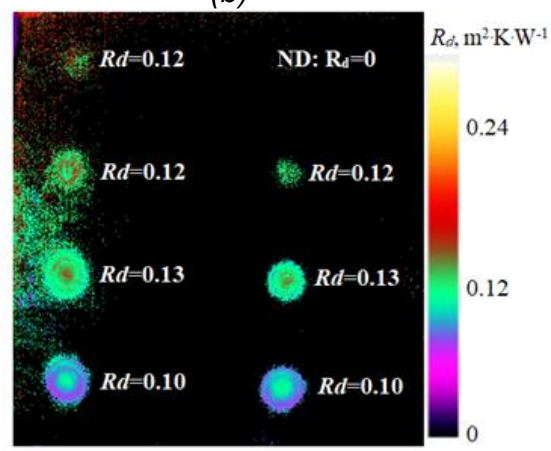

(d)

Fig. 4: Results of 1D defect caracterization by using analytical approach (a-"optimal" raw image, b-image of running contrast, c-depthgram, d-image of defect thermal resistance).

\subsection{Neural network approach}

The NN used in this study was realized in Matlab by means of the NNtool package and consisted of 5 hidden layers with the number of neurons being 10,15, 14, 10 and 5 in each layer respectively. The sigmoidal function a $=$ $1 /[1+\operatorname{Exp}(-n)]$, where a varies from 0 to 1 , and $n$ is the feeding value, has been used for activating the NN. Such function is supposed to be optimal for classifying graphical relationships. Respectively, the NN output layer contained a linear activation function. Numan et al. found that the accuracy in characterizing deeper defects improves with a higher number of NN layers [4], therefore, in this study, a criterion for choosing the number of layers was a compromise between the computation time and the accepted accuracy of defect depth evaluation.

The training of the NN was fulfilled by feeding its input with eight groups of data characterizing defect depths and one group of non-defect data inputs, as well as nine groups of targets representing true depth values. Input data was created by using digitized temperature temporal profiles (64 values in each "defect" group and 608 "non-defect" values) 
at some chosen surface points. It is important noting that the sequence used for training/testing was the sequence of the 1-st derivative (TSR method). A special program which was developed within the Matlab graphics editor allowed the identification of chosen $4 \times 4$ pixel points in both defect and non-defect areas, as shown in figure 5 .

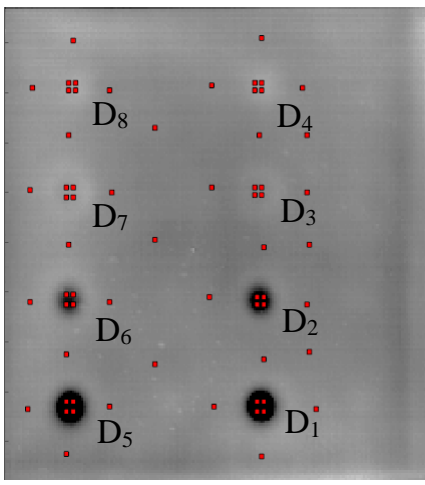

Fig. 5: Location of surface points used for training of NN (images of the first derivative,52 s).

Below we describe a procedure for determining defect size and area by analyzing corresponding defect maps (line-scanning TNDT procedure). Figure 6 shows the normalized raw image, depthgram and map of defects obtained by using the ANN. This approach allows simultaneous determination of defect coordinates and two defect lateral dimensions, as well as defect area and depth. The worst accuracy appeared in estimating defect area (surprisingly up to $300 \%$ in some cases) that can be explained by lateral heat diffusion and peculiarities of the morphological analysis applied. However, the accuracy of estimating defect depth varied from 0 to $40 \%$.

In the raw image (figure 6a), pixel amplitudes were normalized to the range from 0 to 1 with 0 corresponding to sample thickness (defect-free areas). The depthgram produced by the NN (figure 6b) has been normalized using a morphological filter by zeroing those pixels of which size was smaller than a chosen structural element - a circle-shape area with the 5 pixel-radius. Afterwards, the noise values conditioned by defect border effects were removed by applying erosion, and the images were binarized by applying the threshold of 0.1 (figure $6 \mathrm{c}$ ).

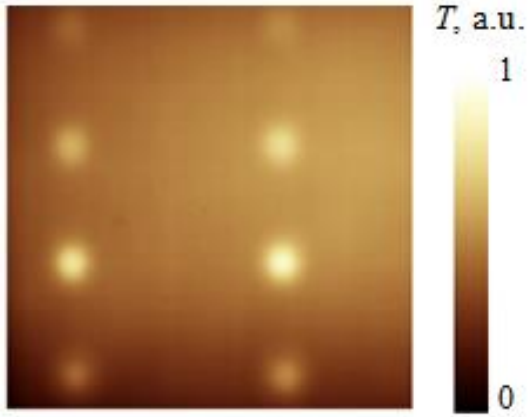

(a)

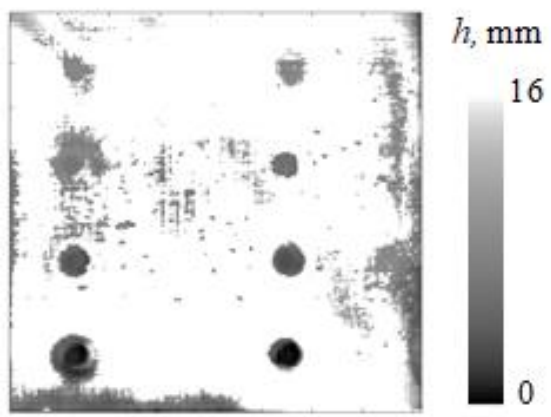

(b)

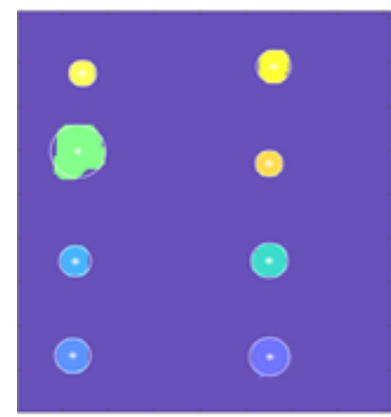

(c)

Fig. 6: Results of 3D defects caracterization using NN approach (a-normalized raw image, b-depthgram, c-binary map of defects).

Table 2 shows the comparison of defects parameters evaluated by both approaches.

Table 2: Characterizing defects in CFRP sample by using analytical formulas (Thermo Fit) and ANN.

\begin{tabular}{|c|c|c|c|c|c|c|c|}
\hline \multirow[b]{2}{*}{ Defect } & \multicolumn{3}{|c|}{ 1D analytical approach } & \multicolumn{4}{|c|}{ Neural network approach } \\
\hline & $\begin{array}{c}\text { Defect } \\
\text { depth, mm } \\
\text { (evaluated/ } \\
\text { true) }\end{array}$ & $\begin{array}{c}\text { Thermal } \\
\text { resistance of } \\
\text { defect } \\
R_{d}, \mathrm{~m}^{2} \cdot \mathrm{K} \cdot \mathrm{W}^{-1}\end{array}$ & $\begin{array}{c}\text { Lateral size of } \\
\text { defect, mm } \\
\text { (evaluated/ } \\
\text { true) }\end{array}$ & $\begin{array}{c}\text { Defect } \\
\text { depth, } \mathrm{mm} \\
\text { (evaluated/ } \\
\text { true) }\end{array}$ & $\begin{array}{l}\text { Diameter of } \\
\text { defect, } \mathrm{mm} \\
\text { (evaluated/ } \\
\text { true) }\end{array}$ & $\begin{array}{l}\text { Literal size of } \\
\text { defect }, \mathrm{mm} \\
\text { (evaluated/ } \\
\text { true) }\end{array}$ & $\begin{array}{c}\text { Coordinates } \\
\text { of defect } \\
\text { location, } \\
\mathrm{mm}\end{array}$ \\
\hline D1 & $0.7 / 1$ & 0.1 & $25 \times 24 / 20 \times 20$ & $0,6 / 1$ & $29 / 20$ & $31 \times 26 / 20 \times 20$ & $26 * 110$ \\
\hline $\mathrm{D} 2$ & $2.9 / 3$ & 0.13 & $20 \times 20 / 20 \times 20$ & $3,0 / 3$ & $23 / 20$ & $25 \times 25 / 20 \times 20$ & $70 * 110$ \\
\hline D3 & $4.9 / 5.5$ & 0.12 & $11 \times 10 / 20 \times 20$ & $5,5 / 5,5$ & $38 / 20$ & $18 \times 18 / 20 \times 20$ & $114 * 110$ \\
\hline D4 & $-{ }^{*} / 7.5$ & 0 & $-* / 20 \times 20$ & $7,1 / 7,5$ & $25 / 20$ & $25 \times 21 / 20 \times 20$ & $158 * 112$ \\
\hline D5 & $0.6 / 0.5$ & 0.1 & $24 \times 23 / 20 \times 20$ & $0,6 / 0,5$ & $28 / 20$ & $26 \times 23 / 20 \times 20$ & $26 \times 25$ \\
\hline D6 & $2.7 / 2.5$ & 0.13 & $26 \times 23 / 20 \times 20$ & $2,7 / 2,5$ & $26 / 20$ & $23 \times 20 / 20 \times 20$ & $70 * 25$ \\
\hline D7 & $4.6 / 5.0$ & 0.12 & $17 \times 15 / 20 \times 20$ & $4,9 / 5,0$ & $20 / 20$ & $41 \times 36 / 20 \times 20$ & $120 * 27$ \\
\hline D8 & $5.8 / 7.0$ & 0.12 & $15 \times 13 / 20 \times 20$ & $7,1 / 7,0$ & $19 / 20$ & $18 \times 18 / 20 \times 20$ & $155^{\star} 29$ \\
\hline
\end{tabular}


The technique of line scanning has demonstrated similar results compared to the classical pulsed TNDT procedure but this technique is more appropriate for automated inspection of large-size objects.

\section{Conclusion}

In this study, we have compared results of using a classical pulsed and line-scanning TNDT procedures in the detection and characterization of defects in a CDRP sample. The defect characterization has been performed by applying simple and robust analytical formulas and an artificial NN. The first approach has allowed producing images of defect depth and thermal resistance, and the NN allowed characterization of defect depth, size, area and location.

\section{Acknowledgments} Program

This study was conducted in the framework of Tomsk Polytechnic University Competitiveness Enhancement

\section{References}

[1] Vavilov V. P. Pawar S. S. Determining the lateral size of subsurface defects during active thermal nondestructive testing.-Russian Journal of Nondestructive Testing, 2016, Vol. 52, №. 9, p. 528-531

[2] Ibarra-Castanedo, C.; Maldague, X. Defect depth retrieval from pulsed phase thermographic data on Plexiglas and aluminum samples. Proc. SPIE 2004, 5405, 348-356.

[3] Munidasa, M.; Mandelis, A.; Ferguson, C. Resolution of photothermal tomographic Imaging of sub-surface defects in metals with ray-optic reconstruction. Appl. Phys. 1992, 54, 244-250

[4] Winfree, W.P.; Plotnikov, Y.A. Defect characterization in composites using a thermal tomography algorithm. In Review of Progress in Quantitative Nondestructive Evaluation; Thompson, D.O., Chimenti, D.E., Eds.; Kluwer Academic Plenum Publishers: Dordrecht, The Netherlands, 1999; Volume 18, pp. 1343-1350.

[5] Toivanen, J.M.; Tarvainen, T.; Huttunen, J.M.J.; Savolainen, T.; Orlande, H.R.B.; Kaipio, J.P.; Kolehmainen, V. 3D thermal tomography with experimental measurement data. Int. J. Heat Mass Transf. 2014, 78, $1126-1134$. [CrossRef]

[6] Sun, J.G. Method for Implementing Depth Deconvolution Algorithm for Enhanced Thermal Tomography 3D Imaging. U.S. Patent No. 8465200, 2013.

[7] Balageas, D.L.; Krapez, J.-C.; Cielo, P. Pulsed photo-thermal modeling of layered materials. J. Appl. Phys. 1986, 59, 348-357. 